๑ Е.А. АРТЮХИНА, И.В. ПРОНИЧЕВА, А.Ш. РЕВИШВИЛИ, 2015

๑ АННАЛЫ АРИТМОЛОГИИ, 2015

УдК 616.126.4-089.168:616.12-008.311

DOI: 10.15275/annaritmol.2015.3.6

\section{УСТРАНЕНИЕ ИНЦИЗИОННЫХ ТАХИКАРДИЙ У ПАЦИЕНТА} С АТРИОФАСЦИКУЛЯРНЫМ ТРАКТОМ ПОСЛЕ ХИРУРГИЧЕСКОЙ ИЗОЛЯЦИИ И ЭЛЕКТРОДЕСТРУКЦИИ \section{АТРИОВЕНТРИКУЛЯРНОГО СОЕДИНЕНИЯ}

Tип статьи: клинический случай

Е.А. Артюхина, И.В. Проничева, А.Ш. Ревишвили

ФГБУ «Научный центр сердечно-сосудистой хирургии им. А.Н. Бакулева» Минздрава России (директор академик РАН и РАМН Л.А. Бокерия); Рублевское шоссе, 135, Москва, 121552, Российская Федерация 
Артюхина Елена Александровна, доктор мед. наук, сердечно-сосудистый хирург; e-mail: artelena.71@mail.ru;

Проничева Ирена Владимировна, канд. мед. наук, кардиолог;

Ревишвили Амиран Шотаевич, доктор мед. наук, профессор, академик РАН, заведующий отделением

Представленный случай демонстрирует успешное устранение трех видов инцизионного трепетания предсердий у пациентки, перенесшей в молодом возрасте операцию изоляции атриовентрикулярного соединения с криодеструкиией проксимального отдела атриовентрикулярного соединения и катетерной электродеструкиией пучка Гиса - по методике, использовавшейся в те годы для лечения некоторых видов синдрома предвозбуждения желудочков. При этом признаки преэкзитации желудочков на ЭКГ сохранялись, но тахикардии у пациентки не отмечалось. В последующем, через 10 лет, зафиксировано атипичное трепетание предсердий, по поводу чего больная поступила в отделение хирургического лечения тахиаритмий для устранения тахиаритмии. С помощью интрейнментстимуляции были выполнены воздействия в области кавотрикуспидального перешейка, а также по латеральной стенке правого предсердия от верхней полой вены до рубца на правом предсердии и от этого рубца до нижней полой вены. Были устранены атипичные трепетания предсердий с длиной иикла 275, 350 и 455 мс.

Катетерная аблация позволяет успешно устранять инцизионные тахиаритмии, при этом проведение по атриофасиикулярному тракту может сохраняться десятилетиями, так как их анатомический субстрат включает элементы нормальной проводящей системы сердиа.

Ключевые слова: атриофасцикулярный тракт; инцизионные аритмии; атипичное трепетание предсердий; радиочастотная аблация; электроимпульсная деструкция.

\title{
ELIMINATION OF INCISIONAL TACHYCARDIAS IN A PATIENT WITH ATRIOFASCICULAR TRACT AFTER SURGICAL ISOLATION AND ELECTRIC DESTRUCTION OF ATRIOVENTRICULAR CONDUCTION
}

\author{
E.A. Artyukhina, I.V. Pronicheva, A.Sh. Revishvili
}

A.N. Bakoulev Scientific Center for Cardiovascular Surgery; Rublevskoe shosse, 135, Moscow, 121552, Russian Federation

Artyukhina Elena Aleksandrovna, MD, PhD, DSc, Cardiovascular Surgeon; e-mail: artelena.71@mail.ru; Pronicheva Irena Vladimirovna, MD, PhD, Cardiologist;

Revishvili Amiran Shotaevich, MD, PhD, DSc, Professor, Academician of Russian Academy of Sciences, Chief of Department

\begin{abstract}
The case of a female patient, 53 years old, shows effective elimination of 3 types of incisional atrial flutters. At young age the patient underwent surgical isolation of atrioventricular (AV) node with cryoablation of the proximal part of AV node conduction and His bundle ablation - according to the technique used at that time for treatment of Wolff-Parkinson-White pattern. On the electrocardiogram the signs of cardiac preexcitation remained, but tachycardia was not reported anymore. After 10 years atypical atrial flutter was reported, so the patient went to the Department of surgical treatment of tachyarrhythmias in Bakoulev Scientific Center for Cardiovascular Surgery to eliminate tachyarrhythmia. Using entrainment pacing a series of radiofrequency influences in the area of caval tricuspid isthmus was performed, as well as on the lateral wall of right atrium from the superior ven a cava to the scar on the right atrium and from the scar to the inferior vena cava. Atypical atrial flutters with a cycle length of 275, 350 and $455 \mathrm{~ms}$ were eliminated.

Catheter ablation can successfully resolve incisional tachycardia, wherein atriofascicular (Mahaim) accessory pathways conduction may remain for decades because their anatomic substrate includes elements of regular conducting system of the heart.

Key words: atriofascicular tract; incisional arrythmias, atypical atrial flutter; radiofrequency catheter ablation; electric pulse destruction.
\end{abstract}

\section{Введение}

B настоящее время катетерная аблация является методом, при помощи которого успешно устраняются почти все виды тахиаритмий, включая аритмии, возникающие у пациентов после операций на открытом сердце.

Однако в 1980-е гг. во всем мире, включая и Россию, для устранения симптоматичных тахи- аритмий использовались техники на открытом сердце, в том числе и с использованием различных видов альтернативной энергии: рассечение эндомиокарда непосредственно скальпелем, криовоздействие, лазерная фотоаблация, электрокоагуляция, а также сочетание этих методов [1]. Кроме того, некоторые аспекты механизмов и субстрата аритмий были недостаточно изучены. Но несмотря на это, использовавшиеся 
хирургические методы и внедрявшиеся в практику катетерные технологии могли избавить пациентов от клинически значимых тахикардий.

Больные, перенесшие операцию на открытом сердце, имеют риск возникновения других нарушений ритма, несмотря на отсутствие исходной аномалии сердца. Важную роль в возникновении аритмий может иметь и электрическое повреждение сердца, связанное, например, с использованием электроимпульсной деструкции проводящей системы, применявшейся в 80-е гг. прошлого столетия.

Представленный случай демонстрирует успешное устранение трех видов инцизионного трепетания предсердий (ТП) у пациентки, перенесшей в молодом возрасте операцию изоляции атриовентрикулярного соединения (АВC) с криодеструкцией проксимального отдела АВС и катетерной электродеструкцией пучка Гиса. Данная методика использовалась в прошлом для лечения некоторых видов синдрома предвозбуждения желудочков.

\section{Клинический случай}

Пациентка X. 53 лет была госпитализирована с жалобами на перебои в работе сердца, ритмичное и неритмичное сердцебиение, учащение ритма до 150 уд/мин.

Из анамнеза. Пациентка считает себя больной с 13 лет, когда ее стали беспокоить приступы учащенного ритмичного сердцебиения, которые купировались вагусными пробами, а в дальнейшем - приемом $\beta$-блокаторов или внутривенно введением новокаинамида. На ЭКГ регистрировалась $\Delta$-волна (рис. 1).

В 1988 г., когда пациентке было 28 лет, она прошла в НЦССХ им. А.Н. Бакулева инвазивное эндокардиальное электрофизиологическое исследование (ЭФИ) по поводу манифестирующего синдрома Вольфа-Паркинсона-Уайта и жалоб на пароксизмальную тахикардию. Выявлено наличие двойных путей атриовентрикулярного (AB) проведения и атриофасцикуляр- ного тракта (АФТ). В условиях искусственного кровообращения и фармакохолодовой кардиоплегии выполнена операция изоляции АВС с криодеструкцией проксимального отдела ABC, а затем в условиях рентгеноперационной - чрезвенозная электроимпульсная деструкция пучка Гиса с целью устранить ретроградное проведение в АВС и исключить возможность формирования риентри тахикардии. В послеоперационном периоде при наличии признаков преэкзитации желудочков на ЭКГ тахикардии у пациентки не отмечалось.

С 1997 г. больную стало беспокоить учащенное ритмичное и аритмичное сердцебиение, прекращавшееся поначалу самостоятельно. С течением времени такие приступы участились, увеличилась их продолжительность, пациентка периодически принимала кордарон, который оказывал положительный эффект. На ЭКГ зафиксировано атипичное ТП.

В ноябре 2011 г. состояние больной ухудшилось, синусовый ритм не восстанавливался. Кордарон принимался на постоянной основе. Пациентка поступила в отделение хирургического лечения тахиаритмий для устранения аритмии.

Проведенное ЭКГ-обследование показало атипичное ТП с частотой сердечных сокращений 90 уд/мин и проведением по дополнительному пути, комплекс $Q R S$ с морфологией полной блокады левой ножки пучка Гиса.

По результатам суточного мониторирования ЭКГ по Холтеру выявлено постоянное атипичное ТП с частотой желудочковых сокращений (ЧЖС) 85-112 уд/мин.

Результаты ЭхоКГ: увеличение полостей сердца; правое предсердие (ПП) увеличено $(4,6 \times 5,3)$ см; трикуспидальный клапан: регургитация II степени; расчетное давление в правом желудочке (ПЖ) - 36 мм рт. ст.; митральный клапан: регургитация II степени; левый желудочек: толщина межжелудочковой перегородки в диастолу $-0,9$ см, конечный систолический размер - 4,6 см, конечный диастолический размер - 6 см, конечный диастолический объем -

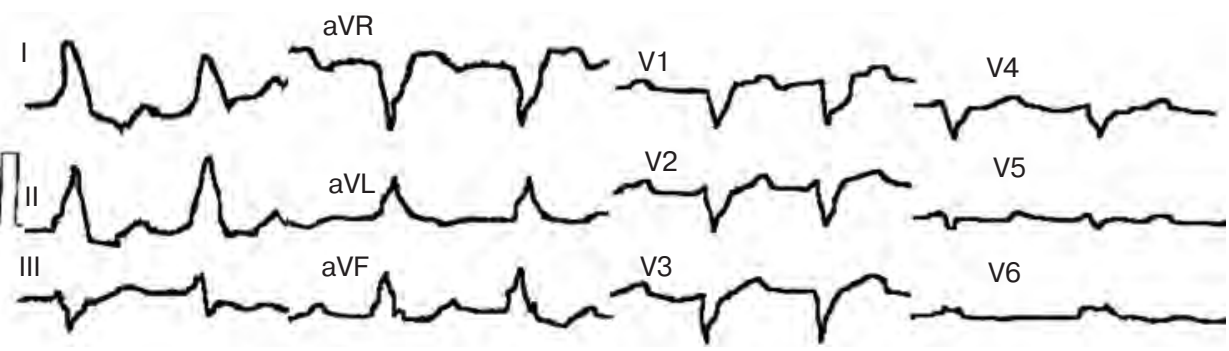

Рис 1. Электрокардиограмма пациентки с атипичным трепетанием предсердий и проведением по атриофасцикулярному тракту 
171 мл, конечный систолический объем - 96 мл, ударный объем - 75 мл, фракция выброса (по формуле Тейхольца) - 44\%.

\section{Протокол радиочастотной аблации (РЧА) и ЭФИ}

Пациентка доставлена в рентгеноперационную на атипичном ТП с ЧЖС 80-120 уд/мин. Под комбинированной анестезией по методике Сельдингера пунктированы правая бедренная и левая подключичная вены. Проведены электроды: 10-полюсный управляемый диагностический в коронарный синус и аблационный холодовой Termo Cool (Biosense Webster, США) в ПП. Длина цикла (ДЦ) ТП - 280 мс с проведением на желудочки 1:1 и 2:1. ЭФИ проводилось на 64-канальном комплексе Prucka Cardiolab 4.0 (General Electric, США).

Выполенена интрейнмент-стимуляция в нескольких отделах ПП. Положительный интрейнмент отмечен в области кавотрикуспидального перешейка. В септальной части перешейка произведена серия радиочастотных воздействий с эффектом удлинения ДЦ до 350 мс и изменением фронта активации. Электрод смещен в область переднелатеральной стенки ПП, где верифицирована зона послеоперационного рубца (разрез на ПП). От этой зоны до нижней полой вены произведена серия линейных воздействий, также с эффектом удлинения ДЦ до $455 \mathrm{мc}$ (рис. 2). Продолжена линия воздействий от зоны рубца до верхней полой вены с эффектом восстановления синусового ритма.

Параметры РЧА: температура $40-44{ }^{\circ} \mathrm{C}$, мощность 38-40 Вт, общее время проведения процедуры - 26 мин. Длительность флюороскопии -18 мин.

При проведении ЭФИ после РЧА зафиксированы ретроградно - вентрикулоатриальная диссоциация, антероградно - проведение по АФТ. Антеградный эффективный рефрактерный период АФТ - 280 мс, антеградная точка Венкебаха АФТ - 300 мс. После введения 500 мг новокаинамида отмечалось временное прекращение антеградного проведения по АФТ с появлением узлового ритма с ЧЖС 38-40 уд/мин. Частой и сверхчастой стимуляцией нарушений ритма не индуцировалось.

Послеоперационный период протекал без осложнений. Пациентка выписана на синусовом ритме, рекомендован обязательный прием $\beta$-блокаторов и антикоагулянтов в течение 3-6 мес.

\section{Обсуждение}

Изучение нодовентрикулярных трактов началось в далеком 1937 г., когда I. Mahaim и A. Bennett впервые описали существование островков проводящей ткани, распространяющихся от АВ-узла к миокарду желудочков [2].

При их дальнейшем исследовании стало очевидно, что большинство трактов в реальности берут начало не в АВ-узле, а в стенке ПП и заканчиваются в правой ножке пучка Гиса, то есть это не нодовентрикулярные и/или нодофасцикулярные волокна, как предполагали ранее, а АВ-тракты и/или АФТ [3]. Проведение импульса по АФТ обеспечивает опережающее возбуждение ПЖ и, следовательно, формирование в период приступа тахикардии комплексов $Q R S$ в виде блокады левой ножки пучка Гиса с отклонением электрической оси $Q R S$ влево. Потенциал Н на электрограмме пучка Гиса не предшествует тахикардическим комплексам $Q R S$, что указывает на антероградное движение импульса в обход ствола пучка Гиса по АФТ к правому желудочку. Ретроградное проведение осушествляется через систему Гиса-Пуркинье к АВ-узлу, где замыкается круг риентри. А значит, предсердия не всегда включаются в этот круг, что исключает появление инвертированных волн $P^{\prime}$ [4].

Первые операции по пересечению волокон Махейма у некоторых больных, страдающих приступами характерной реципрокной тахикардии, подтвердили именно такое расположение [5]. Учитывая, что ретроградным проведением при антидромной тахикардии, связанной с АФТ, являются быстрые пути проведения, устранение проведения по быстрым путям успешно устраняло тахикардию. При этом проведение по АФТ сохранялось [6].

В последующем были описаны различные варианты механизмов тахикардий с участием волокон Махейма, что обусловило сложности хирургического лечения данного вида аритмий. Были также представлены случаи электрической деструкции волокон на открытом сердце [7, 8].

С развитием методики катетерной деструкции субстратов аритмий, впервые использованной в 1978 г. доктором J. Vedel, была предпринята попытка по аблации пучка Гиса [9]. Но данная методика не получила широкого распространения в силу ограничения, связанного с высоким риском имплантации искусственного водителя ритма. В дальнейшем, после того как M. Haissaguerre et al. успешно применили трансвенозную 
катетерную фульгурацию у больных с АФС, она стала использоваться шире [10].

В НЦССХ им А.Н. Бакулева Л.А. Бокерия и А.Ш. Ревишвили впервые использовали открытые методики по устранению истинных и псевдоволокон Махейма (в условиях нормотермического искусственного кровообращения) при постоянной индукции тахикардии путем программированной стимуляции. После вскрытия ПП рассекался миокард в области

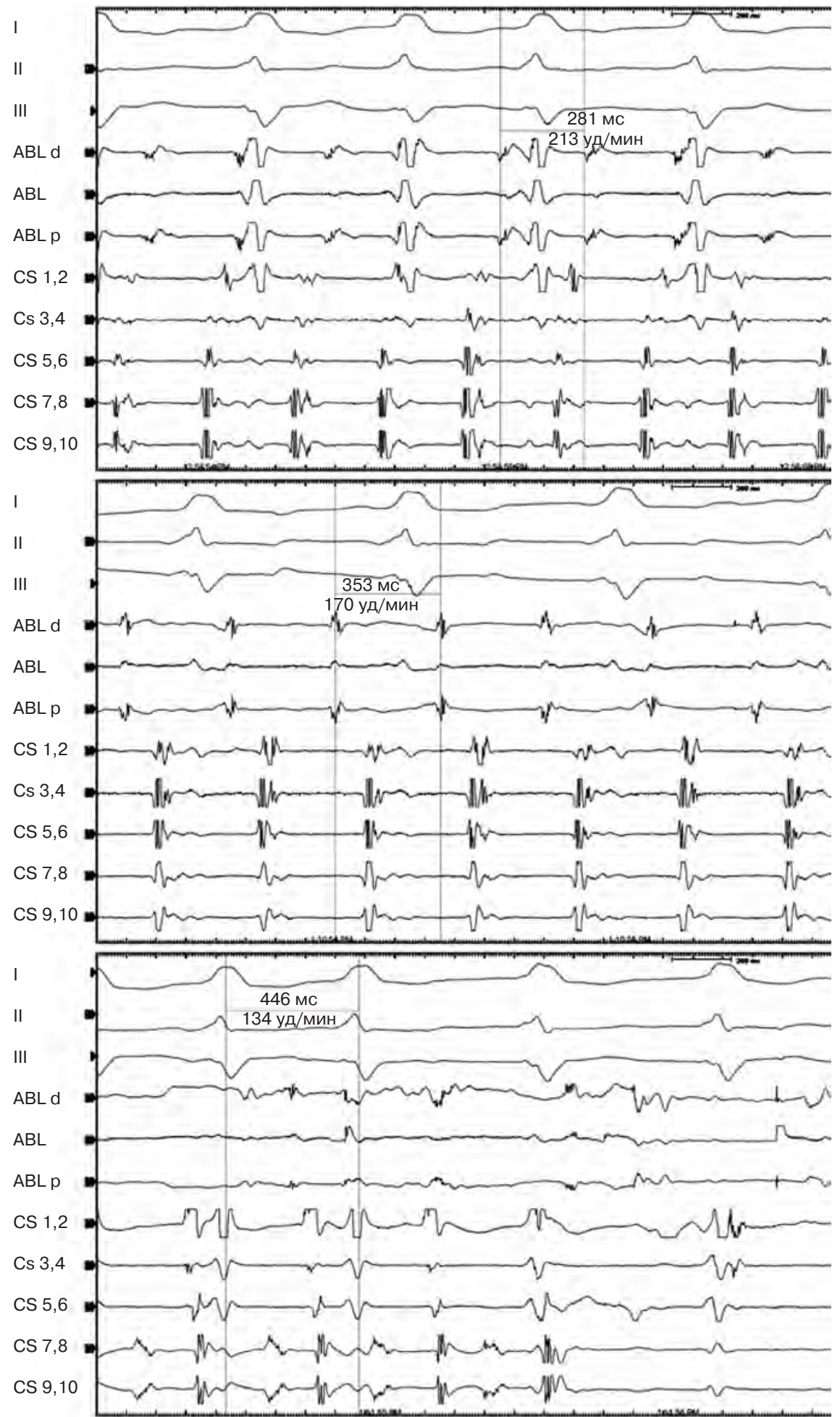

Рис. 2. Электрограммы трех видов атипичного трепетания предсердий: $a-$ с длиной цикла 281 мс; $\sigma$ - с длиной цикла 353 мс; $в$ - с длиной цикла 446 мс.

I, II, III - отведения ЭКГ; ABL - электрограмма с аблационного электрода; CS - электрограмма с электрода, размещенного в коронарном синусе 


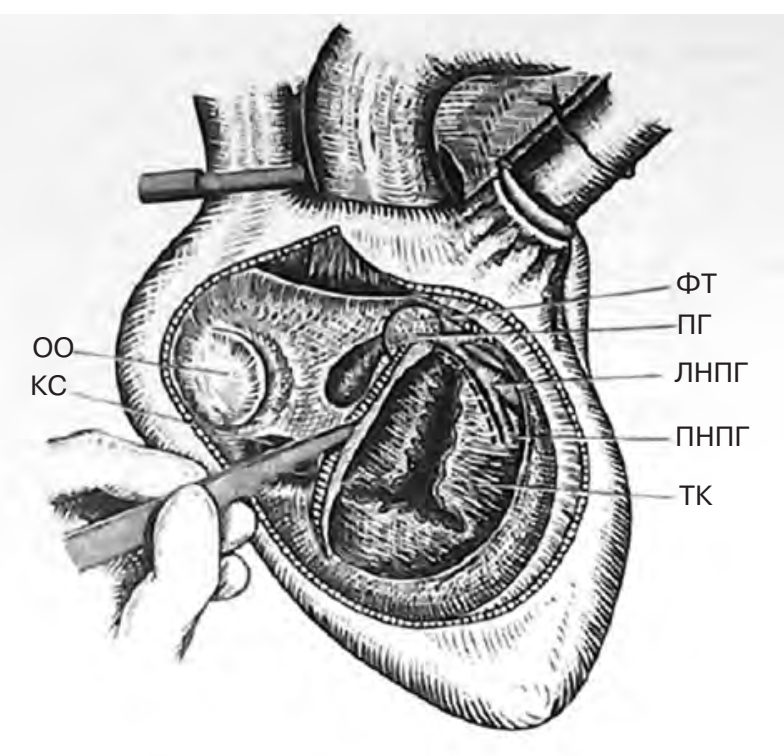

Рис. 3. Операция изоляции атриовентрикулярного соединения (Л.А. Бокерия, А.Ш. Ревишвили, К.А. Абдраманов, 1993 г.).

ОО - овальное окно; КС - коронарный синус; ФТ фиброзный треугольник; ПГ - пучок Гиса; ЛНПГ и ПНПГ - левая и правая ножки пучка Гиса; ТК трехстворчатый клапан

треугольника Коха. Дозированной электрокоагуляцией отсепаровывали тело узла, не повреждая его. Под основанием предсердно-желудочкового узла и вдоль нижнего края пучка Гиса и его правой ножки надсекали эндомиокард. После этого проводили криовоздействие при температуре $-60{ }^{\circ} \mathrm{C}$ в области центрального фиброзного тела (рис. 3) [1].

В дальнейшем, с усовершенствованием катетерных методик, была доказана эффективность именно катетерной аблации [11]. А в настоящее время разработано множество различных техник устранения предсердно-желудочковых соединений, включая АФТ, - конвекционные, холодовые, криоаблация, в ряде случаев используется эпикардиальный доступ [12-14]. Описаны случаи успешного устранения АФТ и у пациентов с врожденными пороками сердца, например с аномалией Эбштейна, и у пациентов детского возраста. С течением времени изменились также и представления об электрофизиологических свойствах трактов Махейма.

\section{Заключение}

Инцизионные аритмии, возникающие у пациента в отдаленном послеоперационном периоде, связаны не только с хирургическими разрезами на ПП, но и с появлением расширенных полостей сердца в результате электротравмы после электроимпульсной деструкции пучка Гиса, а также, вероятно, с постоянной активацией миокарда желудочков не через АВС, а через АФТ даже при отсутствии тахикардии, обусловленной дополнительными путями проведения. Катетерная аблация позволяет успешно устранить инцизионные тахиаритмии, при этом проведение по АФТ может сохраняться десятилетиями, так как их анатомический субстрат включает элементы нормальной проводящей системы сердца.

\section{Конфликт интересов}

Конфликт интересов не заявляется.

\section{Библиографический список}

1. Бокерия Л.А., Ревишвили А.Ш., Абдрахманов К.А. Диагностика и хирургическое лечение тахикардий у больных с нодовентрикулярными волокнами. Грудная и сердечнососудистая хирургия. 1993; 1: 9-14.

2. Mahaim I., Bennett A. Nouvelle recherches surles connexions superieures de la branche gauche du faiscesu de His-Tawara avec cloison interventriculaire. Cardiologia. 1938; 1: 61-7.

3. Tchou P., Lehmann M.H., Jazayeri M., Akhtar M. Atriofascicular connection or a nodoventricular Mahaim fiber? Electrophysiologic elucidation of the pathway and associated reentrant circuit. Circulation. 1988; 77: 837-48.

4. Haissaguerre M., Cauchemez B., Marcus F. Characteristics of the ventricular insertion sites of accessory pathways with anterograde decremental conduction properties. Circulation. 1995; 91: 1077-85.

5. Klein G.J., Guiraudon G.M., Kerr C.R., Sharma A.D., Yee R., Szabo T., Yeung J.A.Y. 'Nodoventricular' accessory pathway: evidence for a distinct accessory atrioventricular pathway with atrioventricular node-like properties. JACC. 1988; 11: 1035-40.

6. Kuck K., Blackburn M., Ouyang F. et al. Retrograde accessory pathway potential recording through a novel quadripolar-tip electrode ablation catheter. Eur. J. Cardiac Pacing Electrophysiol. 1996; 6 (1): 64.

7. Джонсон Д. Предсердные тахикардии и нодовентрикулярные волокна. Кардиология. 1990; 3 (11): 60-2.

8. Guiraudon C.M., Guiraudon G.M., Klein G.J. Histologic evidence for an accessory atrioventricular pathway with AV-nodelike morphology. Circulation. 1988; 78 (Suppl. 2): 40.

9. Bhandari A., Morady F., Shen E.N., Schwartz A.B., Botvinick E., Scheinman M.M. Catheter-induced His bundle ablation in a patient with reentrant tachycardia associated with a nodoventricular tract. JACC. 1984; 4 (3): 611.

10. Haissaguerre M., Warin J.F., Le Metayer P., Maraud L., de Roy L., Montserrat P., Massiere J.P. Catheter ablation of Mahaim fibers with preservation of atrioventricular nodal conduction. Circulation. 1990; 82 (2): 418.

11. Klein L.S., Hackett F.K., Zipes D.P., Miles W.M. Radiofrequency catheter ablation of Mahaim fibers at the tricuspid annulus. Circulation. 1993; 87: 738-47.

12. McClelland J.H., Wang X., Beckman K.J., Hazlitt H.A., Prior M.I., Nakagawa H. et al. Radiofrequency catheter ablation of right atriofascicular (Mahaim) accessory pathways guided by accessory pathway activation potentials. Circulation. 1994; 89: 2655-66.

13. Braun E., Siebbels J., Volkmer M., Ouyang F., Hebe J., Willems S. et al. Radiofrequency-induced preexcited automatic rhythm during ablation of accessory pathways with Mahaimtype preexcitation: does it predict clinical outcome? PACE. 1997; 20: 1124.

14. Филатов А.Г., Голухова Е.3., Рябинина М.Н., Ковалев А.С. Случай успешной радиочастотной аблации атипичного 
левопредсердного трепетания с использованием системы трехмерной нефлюороскопической навигации SJM EnSite Velocity. Креативная кардиология. 2014; 2: 60-70.

\section{References}

1. Bockeria L.A., Revishvili A.Sh., Abdrakhmanov K.A. Diagnosis and surgical treatment of tachycardias in patients with nodoventricular fibers. Grudnaya i serdechno-sosudistaya khirurgiya. 1993; 1: 9-14 (in Russian).

2. Mahaim I., Bennett A. Nouvelle recherches surles connexions superieures de la branche gauche du faiscesu de His-Tawara avec cloison interventriculaire. Cardiologia. 1938; 1: 61-7.

3. Tchou P., Lehmann M.H., Jazayeri M., Akhtar M. Atriofascicular connection or a nodoventricular Mahaim fiber? Electrophysiologic elucidation of the pathway and associated reentrant circuit. Circulation. 1988; 77: 837-48.

4. Haissaguerre M., Cauchemez B., Marcus F. Characteristics of the ventricular insertion sites of accessory pathways with anterograde decremental conduction properties. Circulation. 1995; 91: 1077-85.

5. Klein G.J., Guiraudon G.M., Kerr C.R., Sharma A.D., Yee R., Szabo T., Yeung J.A.Y. 'Nodoventricular' accessory pathway: evidence for a distinct accessory atrioventricular pathway with atrioventricular node-like properties. JACC. 1988; 11: 1035-40.

6. Kuck K., Blackburn M., Ouyang F. et al. Retrograde accessory pathway potential recording through a novel quadripolar-tip electrode ablation catheter. Eur. J. Cardiac Pacing Electrophysiol. 1996; 6 (1): 64 .
7. Johnson D. Atrial tachycardias and node ventricular fibers. Kardiologiya. 1990; 3 (11): 60-2 (in Russian).

8. Guiraudon C.M., Guiraudon G.M., Klein G.J. Histologic evidence for an accessory atrioventricular pathway with AV-nodelike morphology. Circulation. 1988; 78 (Suppl. 2): 40.

9. Bhandari A., Morady F., Shen E.N., Schwartz A.B., Botvinick E., Scheinman M.M. Catheter-induced His bundle ablation in a patient with reentrant tachycardia associated with a nodoventricular tract. JACC. 1984; 4 (3): 611.

10. Haissaguerre M., Warin J.F., Le Metayer P., Maraud L., de Roy L., Montserrat P., Massiere J.P. Catheter ablation of Mahaim fibers with preservation of atrioventricular nodal conduction. Circulation. 1990; 82 (2): 418.

11. Klein L.S., Hackett F.K., Zipes D.P., Miles W.M. Radiofrequency catheter ablation of Mahaim fibers at the tricuspid annulus. Circulation. 1993; 87: 738-47.

12. McClelland J.H., Wang X., Beckman K.J., Hazlitt H.A., Prior M.I., Nakagawa H. et al. Radiofrequency catheter ablation of right atriofascicular (Mahaim) accessory pathways guided by accessory pathway activation potentials. Circulation. 1994; 89: 2655-66.

13. Braun E., Siebbels J., Volkmer M., Ouyang F., Hebe J., Willems S. et al. Radiofrequency-induced preexcited automatic rhythm during ablation of accessory pathways with Mahaimtype preexcitation: does it predict clinical outcome? PACE. 1997; 20: 1124

14. Filatov A.G., Golukhova E.Z., Ryabinina M.N., Kovalev A.S. Successful radiofrequency ablation of atypical left atrial flutter via threedimensional nonfluoroscopic navigational system SJM EnSite Velocity: a case report. Kreativnaya kardiologiya. 2014; 2: 60-70 (in Russian). 